\title{
Body mass index was linked with multi-cardiometabolic abnormalities in Chinese children and adolescents: a community-based survey
}

\author{
Huijing He${ }^{1}$, Li Pan ${ }^{1}$, Jianwei Du², Yuming Jin², Pengben Jia² and Guangliang Shan ${ }^{\text {** }}$
}

\begin{abstract}
Background: Evidence on how body mass index (BMI) influence cardiometabolic health remains sparse in Chinese children and adolescents, especially in south China. We aim to investigate the effect of overweight and/or obesity on high blood pressure (HBP), dyslipidemia, elevated serum uric acid (SUA) and their clustering among children and adolescents in an island in South China.
\end{abstract}

Methods: Using multi-stage cluster sampling method, 1577 children and adolescents aged 7-18 in Hainan province, south China, participated in the survey. The association between body mass index and cardiometabolic indexes were explored. Overweight and obesity were classified according to criteria of World Health Organization for children and adolescents aged 5 to 19. Restricted cubic spline models were used to examine the possible non-linear association between BMI and cardiometabolic profiles. Multivariable logistic regression models were fitted to examine the effect size of BMI on cardiometabolic disorders including HBP, elevated SUA and dyslipidemia. Comorbidity of at least two cardiometabolic abnormalities (HBP, dyslipidemia, elevated SUA) was defined as clustering of cardiometabolic risk factors.

Results: Comparing with normal weight and underweight subjects, overweight/obese youths had higher levels of BP, SUA, triglyceride, low-density lipoprotein but lower level of high-density lipoprotein. Overweight/obese youth had higher risk of dyslipidemia (OR:2.89, 95\%Cl: 1.65-5.06), HBP (OR:2.813, 95\%Cl: 1.20-6.59) and elevated SUA (OR: 2.493, 95\%Cl: 1.45-4.27), respectively, than their counterparts. The sex-, age-adjusted prevalence of abnormalities clustering was $32.61 \%$ (95\% Cl: $20.95 \%$ to $46.92 \%$ ) in overweight/obesity group, much higher than in the under/normal weight group (8.85\%, 95\%Cl: $7.44 \%$ to $10.48 \%)$.

Conclusion: Excess adiposity increased the risk of elevated serum uric acid, serum lipids, blood pressure and their clustering among children and adolescents in south China.

Keywords: Pediatric, Overweight, Obesity, Cardiometabolic, Epidemiology, Public health

*Correspondence: guangliang_shan@163.com

${ }^{1}$ Department of Epidemiology and Statistics, Institute of Basic Medical Sciences, Chinese Academy of Medical Sciences; School of Basic

Medicine, Peking Union Medical College, 5 Dongdansantiao, Dongcheng District, Beijing 100005, China

Full list of author information is available at the end of the article

\section{Introduction}

Cardiometabolic risk factors in childhood, such as high blood pressure, elevated serum uric acid, dyslipidemia, are associated with earlier onset and greater risk of chronic diseases in adults [1-3].Excess adiposity is associated with childhood metabolic profiles[4], and 
may increase the risk of cardiometabolic abnormalities, such as high blood pressure (HBP), dyslipidemia, insulin resistance, and elevated serum uric acid (SUA) [5-7]. Overweight and obesity in children and adolescents have become a significant public health issue for both developed and developing countries given its fast increasing over the past few decades $[8,9]$. The prevalence of overweight and obesity in Chinese children also continuously increased in the past thirty years $[10,11]$.

Since cardiometabolic disease develops gradually, it is important to identify children and adolescents who are in high risk and have already had abnormalities. There are previous studies exploring the association between excess adiposity and pediatric cardio-metabolic risk factors [12-16], which provided valuable health information, but these health profiles did not take elevated serum uric acid into consideration, and the investigation on comorbidity of multiple cardiometabolic abnormalities is sparse, especially among Chinese youths. Hainan is an island located at the southernmost of China with tropical climate and has long been on the fringe of the Chinese cultural sphere. Limited study has carried out in Hainan partially because of its geographic location. Little was known about the youths' cardiometabolic health profiles in this place. In 2013, we carried out a cross-sectional study among children and adolescents aged 7-18 in Hainan province, with the purpose of exploring the effect of excess body weight on cardiometabolic health profiles in the youth population. Evidence found by this study may provide useful information for early prevention of cardiometabolic risk among children and adolescents.

\section{Methods and materials Study design and population}

The present study is cross-sectional design and based on data collected in Hainan province, which is an island in southernmost China. The sampling method is the same with our previous studies $[17,18]$. Briefly, from Nov to Dec 2013, a multi-stage stratified clustering sampling method was used to enroll participants. In the first stage, the provincial capital city (Haikou), one mid-sized city (Zhanzhou) and two counties (Changjiang and Baisha) were selected based on their economic status measured by local gross domestic product (GDP). In the second stage, districts were selected from urban areas and townships were selected from rural areas. In the last stage, communities were selected from districts and villages were selected from townships. The inclusion criteria for participants were: aged 7-18 years; lived in current residence for at least one year. The exclusion criteria were: youths who have been diagnosed as high blood pressure, type 1 diabetes or took medication for these diseases. Ethical approval was obtained from the Bioethical Committee of Institute of Basic Medical Sciences, Chinese Academy of Medical Sciences. Informed consent was obtained from parents/guardians of the participants.

As in this study, several cardiometabolic disorders were measured, we used the lowest prevalence among these disorders to ensure the statistical power. Based on our previous analysis, the prevalence of dyslipidemia and high blood pressure among children and adolescents were $20 \%$ and $7 \%[17,18]$, respectively. As there were no standard definition of elevated serum uric acid in children and adolescents, we used the prevalence of high blood pressure to calculate the sample size. The following formula was used:

$$
n=Z_{\alpha}^{2} \times p q / d^{2}
$$

Alpha $(\alpha)$ was the significant level, p was the prevalence of HBP, $q$ equals $1-p$, and $d$ was the error tolerance. To reach a significant level of 0.05 and error tolerance $0.2 \times p$, the estimated minimum sample size was 1276 . An additional $20 \%$ to the minimum sample size was added factoring in possible non-compliance rate and targeted 1532 subjects. Finally, 1609 children and adolescents participated in the study.

\section{Measurements}

A standardized questionnaire interview was conducted face-to-face by fixed trained staff to collect information on demographic characteristics at community health centers or village clinics in the study sites. Height was measured to the nearest $0.1 \mathrm{~cm}$ using a fixed stadiometer by the same staff to avoid measurement system error. Body weight were measured barefooted with light clothes using a body composition analyzer (TANITA BC-420, Japan) with decimal accuracy. BMI was calculated as weight in kilograms divided by the square of height in meters $\left(\mathrm{kg} / \mathrm{m}^{2}\right)$. Using a digital blood pressure measuring device (Omron HEM-907, Japan) with children customized cuff size, systolic blood pressure (SBP) and diastolic blood pressure (DBP) were measured on the right arm of individual after at least 5 mins in a sitting position in the study morning. The average value of the three measures were recorded. A $9 \mathrm{ml}$ (at least $8 \mathrm{~h}$ fasting overnight) venous blood sample from each participant was draw by qualified nurses for serum lipids and uric acid tests by Chemistry Analyzer (ROCHE Cobas8000C701, USA). For each day of the survey, the samples were kept in a portable, insulated cool box with ice packs to maintain their temperature at $0-4{ }^{\circ} \mathrm{C}$ for up to $3 \mathrm{~h}$ before being transported to the laboratory of local center for disease control and prevention (CDC) for immediate processing. Serum lipid tests included total cholesterol (TC), 
triglycerides (TG), high-density lipoprotein cholesterol (HDL-C) and low-density lipoprotein cholesterol (LDL-C).

\section{Definitions}

Underweight, normal weight, overweight and obesity were classified according to criteria of World Health Organization (WHO) for children and adolescents aged 5 to 19[19], which were: BMI-for-age lower than two standard deviations (SDs) below the WHO Growth Reference median as underweight; greater than one SD as overweight and greater than two SDs as obesity.

Serum lipid disorders were defined according to the National Heart, Lung, and Blood Institute (NHLBI) cholesterol screening guidelines and cut points backed by the American Academy of Pediatrics [20], and has been described in detail by our previous study [18].

Blood pressure was classified into normal BP and high blood pressure based on the updated guidelines of the Fourth Report on the Diagnosis, Evaluation, and Treatment of High Blood Pressure in Children and Adolescents [21]. As there is no universally accepted definition of childhood hyperuricemia, we use $5.7 \mathrm{mg} / \mathrm{dl}(339 \mu \mathrm{mol} / \mathrm{l})$ as the criteria for elevated serum uric acid because in the National Health and Nutrition Examination Survey (NHANES) population, higher than $5.7 \mathrm{mg} / \mathrm{dl}$ increased the risk of metabolic syndrome in youths [22].

Comorbidity of at least two cardiometabolic abnormalities (HBP, dyslipidemia, elevated SUA) was defined as clustering of cardiometabolic risk factors.

\section{Statistical analyses}

After excluding subjects with missing data on main risk factors (height, weight, blood pressure, serum uric acid, serum lipids), data on 1577 youths were analyzed.

Summary results were presented as mean (SD) for normally distributed continuous data, median (interquartile range, IQR) for continuous data in-normally distributed, and counts (percentage, \%) for categorical data. T-test (for normally distributed data) or U MannWhitsney test (for non-normally distributed data) was used to compare continuous data between two groups. Chi-square test was used to compare grouped data. Twoway analysis of covariance (for normally distributed data) or quantile regression models (for non-normally distributed data) were used to compare cardiometabolic profiles among body weight groups after adjusted for potential confounders. Partial correlation analysis was performed to understand the correlation between BMI and cardiometabolic indexes by adjusting potential confounders. Scatter plots were presented to show these correlations. Factors that were associated with both BMI and cardiometabolic indexes, such as age and residential areas, were set as covariates in the adjusted regression models, to avoid the potential confounding caused by these factors.

Logistic regression models were used to calculate the multi-variable adjusted prevalence of elevated SUA, HBP and dyslipidemia among different body weight groups [23]. Restricted cubic spline (RCS) models were used to examine the possible non-linear association between BMI and cardiometabolic variables. Tests for non-linearity used the likelihood ratio test, comparing the model with only the linear term to the model with linear and the cubic spline terms [24]. Logistic regression models were fitted to examine the effect size of BMI on cardiometabolic disorders including high blood pressure, elevated SUA and dyslipidemia.

A $p$-value $<0.05$ (two-tailed) was considered statistically significant. Because of the limited number of obesity in both sexes, obesity was combined with overweight as one group, presented as overweight/obesity in the present study. All statistical procedures were performed using SAS 9.4 (SAS Institute Inc. Cary, NC, USA).

\section{Results}

Basic characteristics and cardiometabolic indexes

The demographic and basic clinical information were presented in Table 1. The mean age of the whole participants was $13.59 \pm 3.25$, and $13.25 \pm 3.06$ in boys, $13.84 \pm 3.37$ in girls. Boys and girls were different in age distribution, height, weight, SUA, blood pressure and serum lipids (all $\mathrm{p}$ values less than 0.05$)$.

The association between BMI and cardiometabolic indexes The correlation between BMI and SUA, blood pressure and serum lipids, stratified by sex, were presented as scatter plots in Fig. 1, and their partial correlation coefficients were given. All cardiometabolic indexes were significantly correlated with BMI, with p values less than 0.01 in both sexes, but the effect size of coefficients varied greatly. The correlations between BMI and SBP seemed stronger than DBP in both sexes (boys: $r=0.256$ for SBP and 0.117 for DBP; girls: $r=0.200$ for SBP and 0.040 for DBP). For boys, the correlation between BMI and SUA seemed stronger than other indexes $(r=0.315)$, but this correlation seemed weaker in girls $(r=0.154)$.

The comparison between underweight, normal weight and overweight/obesity on SUA, blood pressure and serum lipids were presented in Table 2. After adjusted for age and residential areas, the overweight/obesity group had higher SUA in both sexes ( $\mathrm{p}=0.016$ in boys; $p<0.001$ in girls). For blood pressure, only in girls, the overweight/ obesity group had higher SBP $(p<0.001)$. No difference was tested in DBP. In both boys and girls, overweight/ obesity group had higher level of TG, LDL-C, but lower HDL-C than their counterparts $(P<0.05)$. 
Table1 Basic characteristics of children and adolescents aged 7-18 years in Hainan Province, China, 2014

\begin{tabular}{|c|c|c|c|c|c|c|c|}
\hline \multirow{2}{*}{$\begin{array}{l}\text { Characteristics } \\
\text { Age (year, mean, SD) }\end{array}$} & \multicolumn{2}{|c|}{ Boys $(n=666)$} & \multicolumn{2}{|c|}{ Girls $(n=911)$} & \multicolumn{2}{|c|}{ Total $(n=1577)$} & \multirow{2}{*}{$\begin{array}{l}P \\
<0.001\end{array}$} \\
\hline & 13.25 & 3.06 & 13.84 & 3.37 & 13.59 & 3.25 & \\
\hline \multicolumn{8}{|l|}{ Age groups (n, \%) } \\
\hline $7-8$ & 43 & 6.46 & 67 & 7.35 & 110 & 6.98 & \multirow[t]{6}{*}{$<0.001$} \\
\hline $9-10$ & 146 & 21.92 & 181 & 19.87 & 327 & 20.74 & \\
\hline $11-12$ & 159 & 23.87 & 154 & 16.90 & 313 & 19.85 & \\
\hline $13-14$ & 118 & 17.72 & 103 & 11.31 & 221 & 14.01 & \\
\hline $15-16$ & 89 & 13.36 & 178 & 19.54 & 267 & 16.93 & \\
\hline $17-18$ & 111 & 16.67 & 228 & 25.03 & 339 & 21.50 & \\
\hline Urban (n, \%) & 379 & 56.91 & 559 & 61.36 & 938 & 59.48 & \multirow[t]{2}{*}{0.075} \\
\hline Rural $(\mathrm{n}, \%)$ & 287 & 43.09 & 352 & 38.64 & 639 & 40.52 & \\
\hline Height (cm) & 150.42 & 15.69 & 148.05 & 12.11 & 149.05 & 13.78 & 0.001 \\
\hline Weight (kg) & 39.61 & 12.48 & 38.16 & 9.94 & 38.77 & 11.10 & 0.013 \\
\hline BMI $\left(\mathrm{kg} / \mathrm{m}^{2}\right.$, mean, SD) & 17.02 & 2.68 & 17.06 & 2.52 & 17.04 & 2.58 & 0.718 \\
\hline Uric acid ( $\mu$ mol/L, median, IQR) & 348.15 & 129.20 & 294.70 & 83.70 & 314.00 & 104.80 & $<0.001$ \\
\hline $\mathrm{SBP}(\mathrm{mmHg}$, mean, SD) & 108.56 & 12.51 & 104.53 & 10.49 & 106.23 & 11.56 & $<0.001$ \\
\hline DBP (mmHg, mean, SD) & 65.50 & 8.52 & 66.84 & 8.23 & 66.27 & 8.37 & 0.001 \\
\hline TC (mmol/L, median, IQR) & 3.98 & 0.95 & 4.17 & 0.94 & 4.09 & 0.92 & $<0.001$ \\
\hline TG (mmol/L, median, IQR) & 0.63 & 0.32 & 0.71 & 0.32 & 0.68 & 0.34 & $<0.001$ \\
\hline LDL-C (mmol/L, median, IQR) & 2.24 & 0.77 & 2.38 & 0.82 & 2.33 & 0.81 & $<0.001$ \\
\hline HDL-C (mmol/L, median, IQR) & 1.42 & 0.42 & 1.46 & 0.39 & 1.44 & 0.39 & 0.043 \\
\hline Creatinine ( $\mu \mathrm{mol} / \mathrm{L}$, median, IQR) & 58.00 & 26.80 & 52.80 & 15.30 & 54.50 & 16.90 & $<0.001$ \\
\hline
\end{tabular}

\section{The association between BMI and cardiometabolic disorders}

The restrict cubic splines demonstrating the relationship between BMI and elevated SUA, HBP and dyslipidemia, after adjusted for age, sex and residential area, were presented in Fig. 2. When took BMI as continuous variable into regression models, only linear positive associations of BMI with elevated SUA $(P<0.001)$ and dyslipidemia $(P<0.001)$ were observed. Neither linear nor non-linear significant association between BMI and HBP was tested by RCS $(P>0.05)$. When took BMI as grouped variable (under/normal weight vs. overweight/obesity) to fit the logistic regression model by sex, increased risk of cardiometabolic disorders in the overweight/obesity group were observed. In the overall model, overweight/obese youth had 1.89, 1,81, and 1.49 times higher risk of dyslipidemia, HBP and elevated SUA, respectively, comparing with the under/normal weight youths (Fig. 2).

Generally, the prevalence of elevated SUA, HBP and dyslipidemia increased with higher BMI classifications. In overall, in underweight, normal weight and overweight/obesity groups, the prevalence of elevated SUA were $32.71 \%, 37.35 \%$ and $58.21 \%$, respectively; the prevalence of HBP were $5.14 \%, 5.48 \%$ and $10.45 \%$, respectively; the prevalence of dyslipidemia were $16.36 \%, 18.52 \%$ and $31.34 \%$, respectively. The sex- and age- adjusted prevalence of elevated SUA, HBP and dyslipidemia in underweight, normal weight and overweight/obesity groups were: $31.81 \%(95 \% \mathrm{CI}: 25.57 \%$ to $38.78 \%)$, $38.29 \%$ (95\%CI: $35.42 \%$ to $41.25 \%$ ) and $60.00 \%$ (95\%CI: $46.87 \%$ to $71.83 \%$ ); $4.93 \%$ (95\%CI: $2.73 \%$ to $8.73 \%$ ), $5.28 \%$ (95\%CI: $4.14 \%$ to $6.72 \%$ ) and $13.46 \%$ (95\%CI: $6.44 \%$ to $26.02 \%) ; 15.74 \%$ (95\%CI: $11.46 \%$ to $21.23 \%$ ), $18.43 \%$ (95\%CI: $16.32 \%$ to $20.74 \%$ ) and $38.99 \%$ (95\%CI: $27.10 \%$ to $52.37 \%$ ).

The sex-specific crude prevalence and adjusted prevalence of elevated SUA, HBP and dyslipidemia in different BMI groups were presented in Fig. 3. Girls had lower prevalence of elevated SUA then boys in underweight and normal weight groups (both adjusted $\mathrm{P}<0.001$ ), but similar prevalence in the overweight/obese group (adjusted $\mathrm{P}=0.4507)$. No other sex difference of cardiometabolic disorders prevalence was found in body weight groups.

\section{Clustering of cardiometabolic abnormalities}

The crude prevalence of the clustering of at least two cardiometabolic abnormalities in under/normal weight and overweight/obesity groups were $9.21 \%$ and $23.88 \%$, respectively (Fig. 4-A). After adjusted for sex, age and residential areas, the prevalence of abnormalities clustering were $32.61 \%$ (95\% CI: $20.95 \%$ to $46.92 \%$ ) in overweight/ 

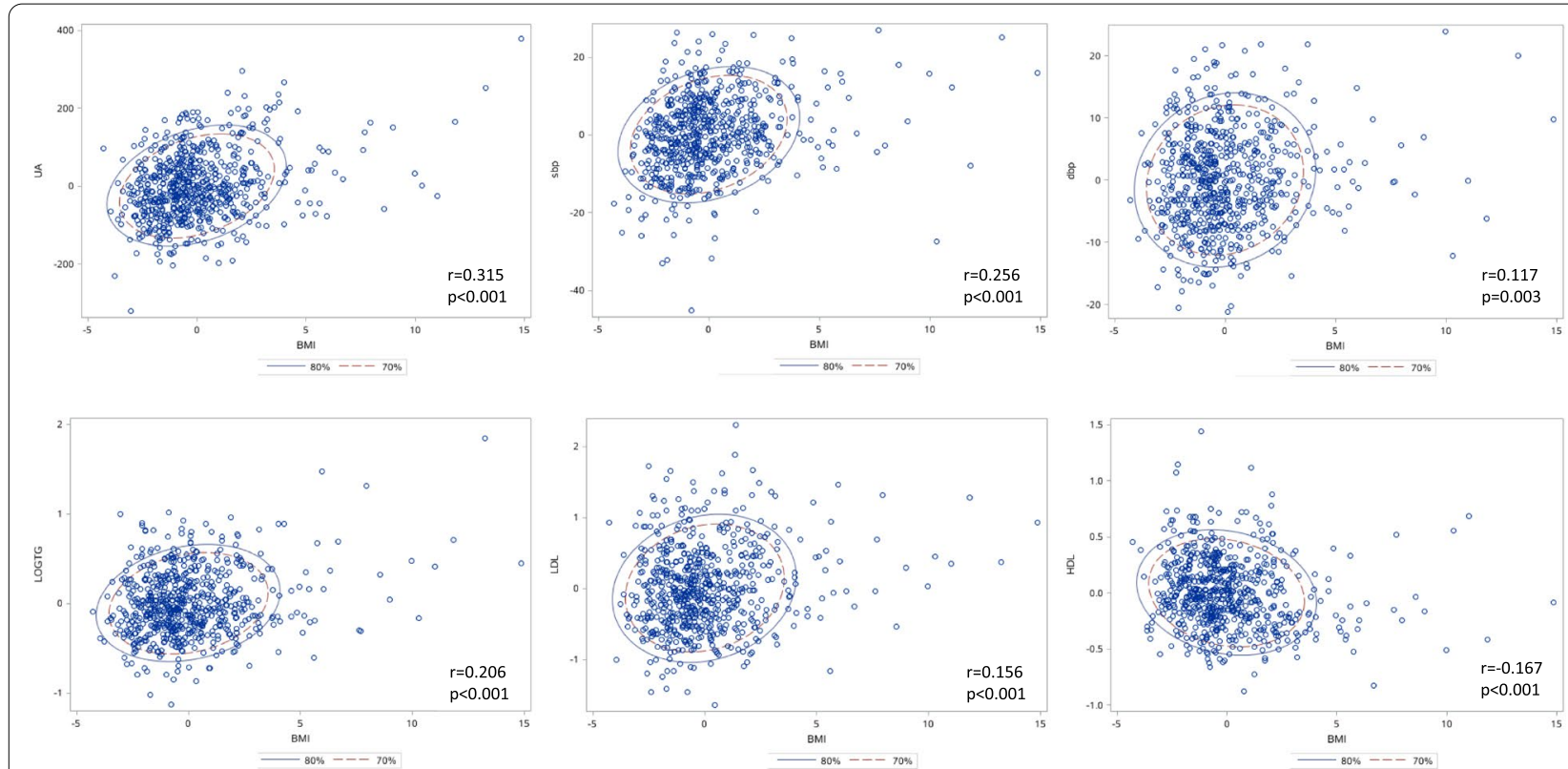

Boys
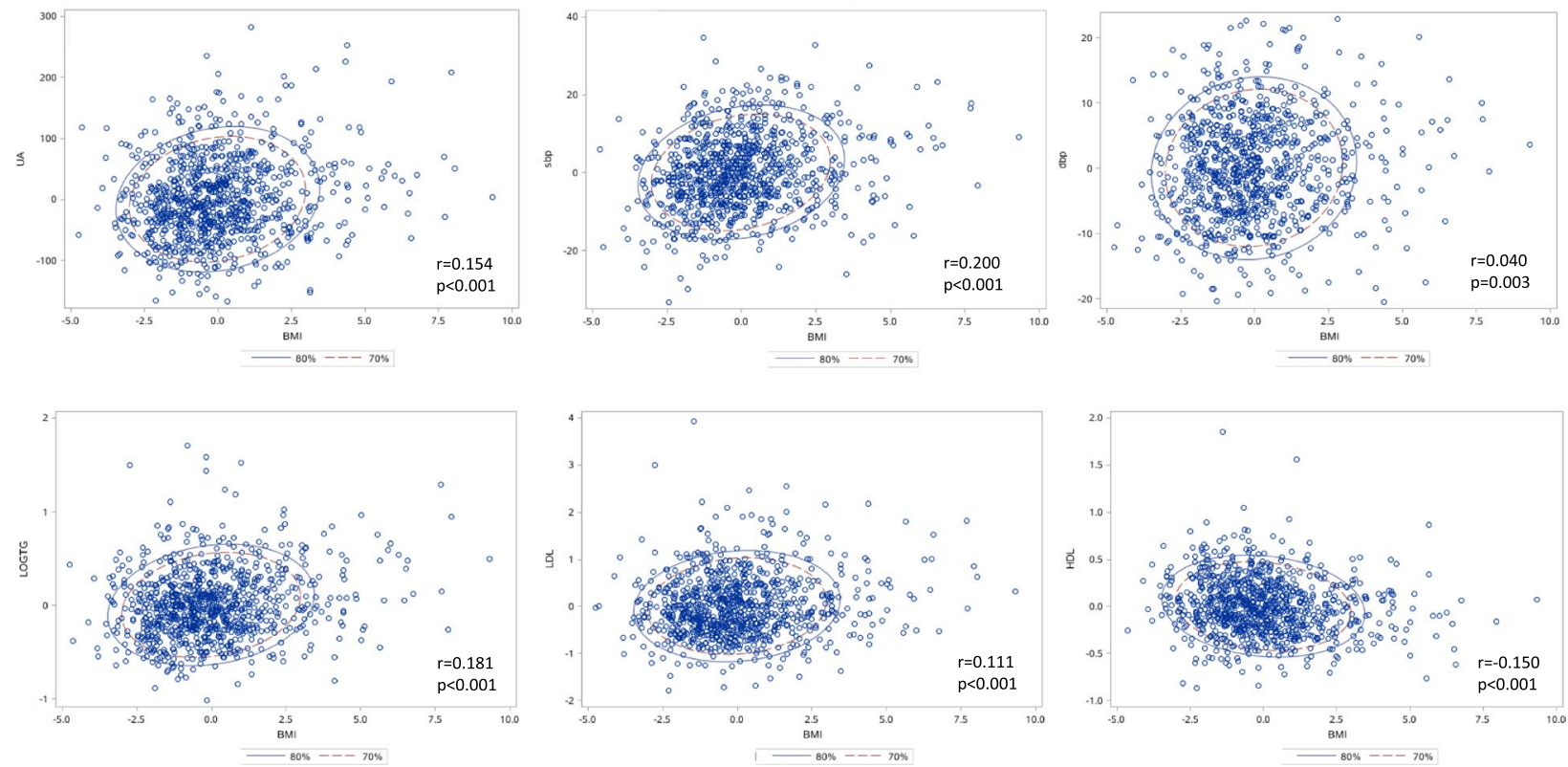

Girls

Fig. 1 The correlation between body mass index and cardiometabolic profiles in separated sex. A Boys; B Girls. The partial correlation coefficients were calculated after adjusted for age and residential areas

obesity group, much higher than in the under/normal weight group (8.85\%, 95\%CI: $7.44 \%$ to $10.48 \%$ ) (Fig. 4 B). The intersection of cardiometabolic abnormalities in the study population were presented as Venn diagrams in Fig. 4-C and D.

\section{Discussion}

In this study, based on a representative population sample, we explored the association between body mass index and cardiometabolic profiles, especially take elevated serum uric acid as one of the components of cardiometabolic abnormalities, in a southernmost island of 


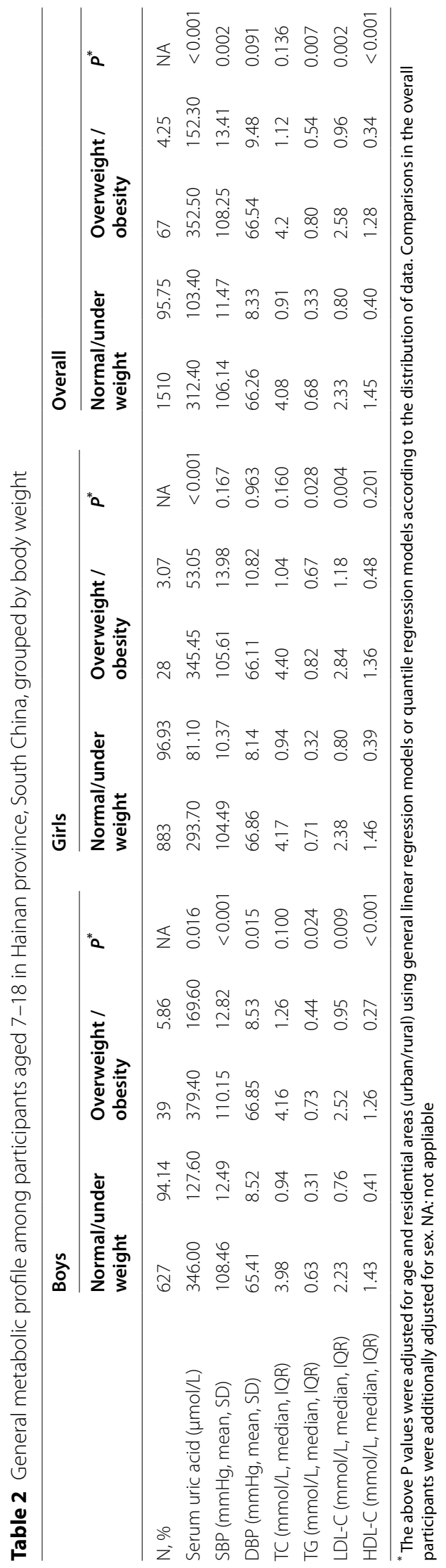




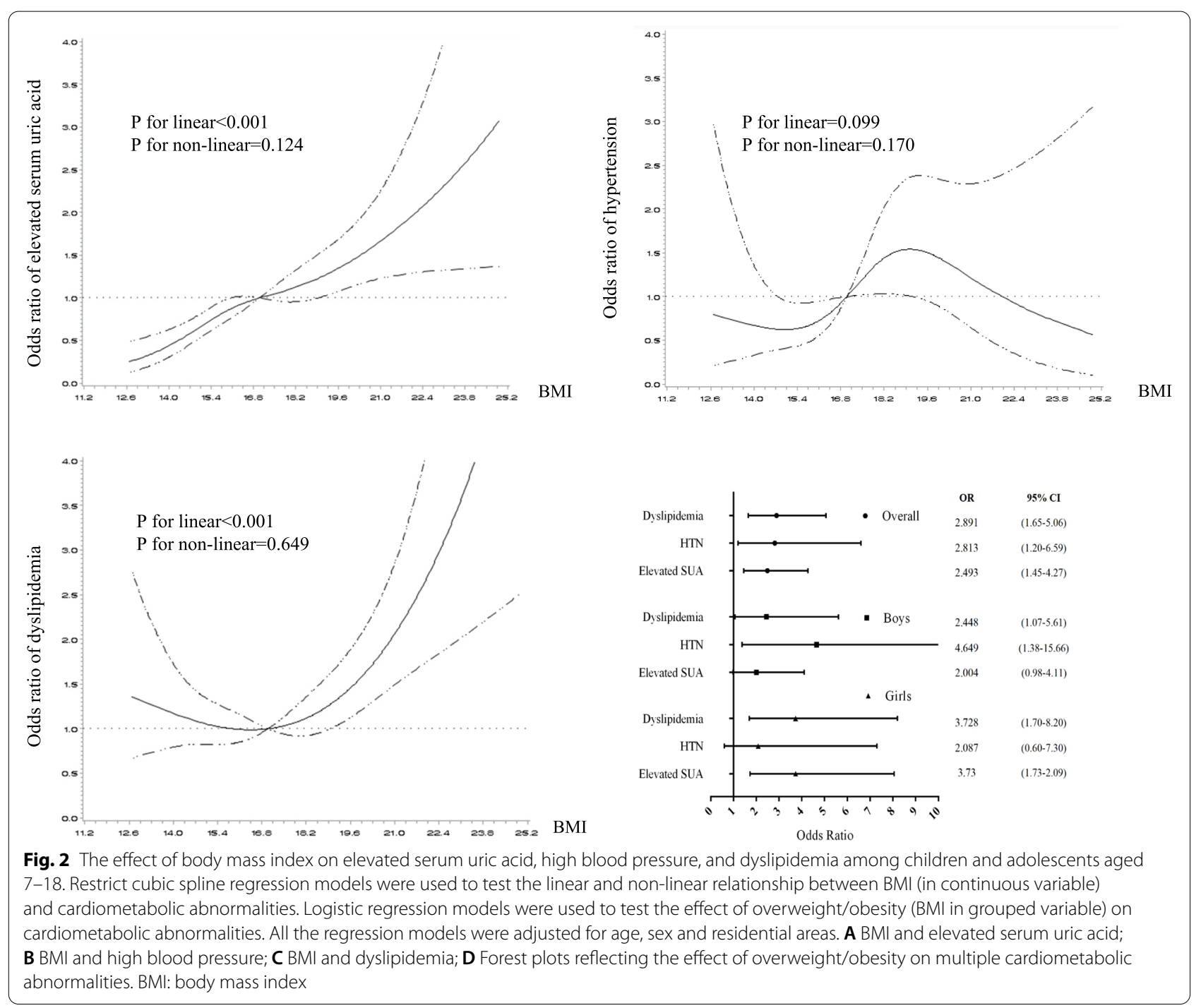

China. Our findings revealed that BMI was linked with multiple cardiometabolic abnormalities and their clustering. The prevalence of cardiometabolic abnormalities increased with elevated BMI, and the level of serum lipids, blood pressure and serum uric acid were associated with overweight and/or obesity regardless of sex.

The epidemic of overweight and obesity is increasing in Chinese children $[10,11]$ and varied greatly by regions. Compared with the population in Northern China, both children and adults in Southern China have a smaller body size [25]. In a national survey, compared with other areas, Hainan has a relatively low prevalence of overweight and obesity [26]. Similar with their finding (2.3\% in Hainan), youths in our study have an overweight/obesity prevalence of $4.25 \%$. The prevalence of childhood cardiometabolic disorders has substantial geographic variations in China. Based on a national school survey, the prevalence of childhood HBP and dyslipidemia were $18.2 \%$ and $15.8 \%$, respectively[27], comparing with $5.9 \%$ and $18.8 \%$ in crude HBP and dyslipidemia prevalence in the current findings. The lower prevalence of HBP in Hainan youth maybe attribute to its tropical weather or dietary patterns [28] which need further exploration.

The relationship between excess adiposity and cardiometabolic diseases in children and adolescents has been acknowledged by previous studies $[12-15,29]$. For example, the Bogalusa Heart Study found that $70 \%$ of obese youths aged $5-17$ had at least one cardiovascular risk factor [30]. Zhang et al. also reported that overweight and/or obesity were associated with increased levels of cardiovascular risk factors among children aged 7.5-13 years in Guangdong, China [14]. Cardiovascular risk factors, such as high blood pressure and dyslipidemia are positively associated with 

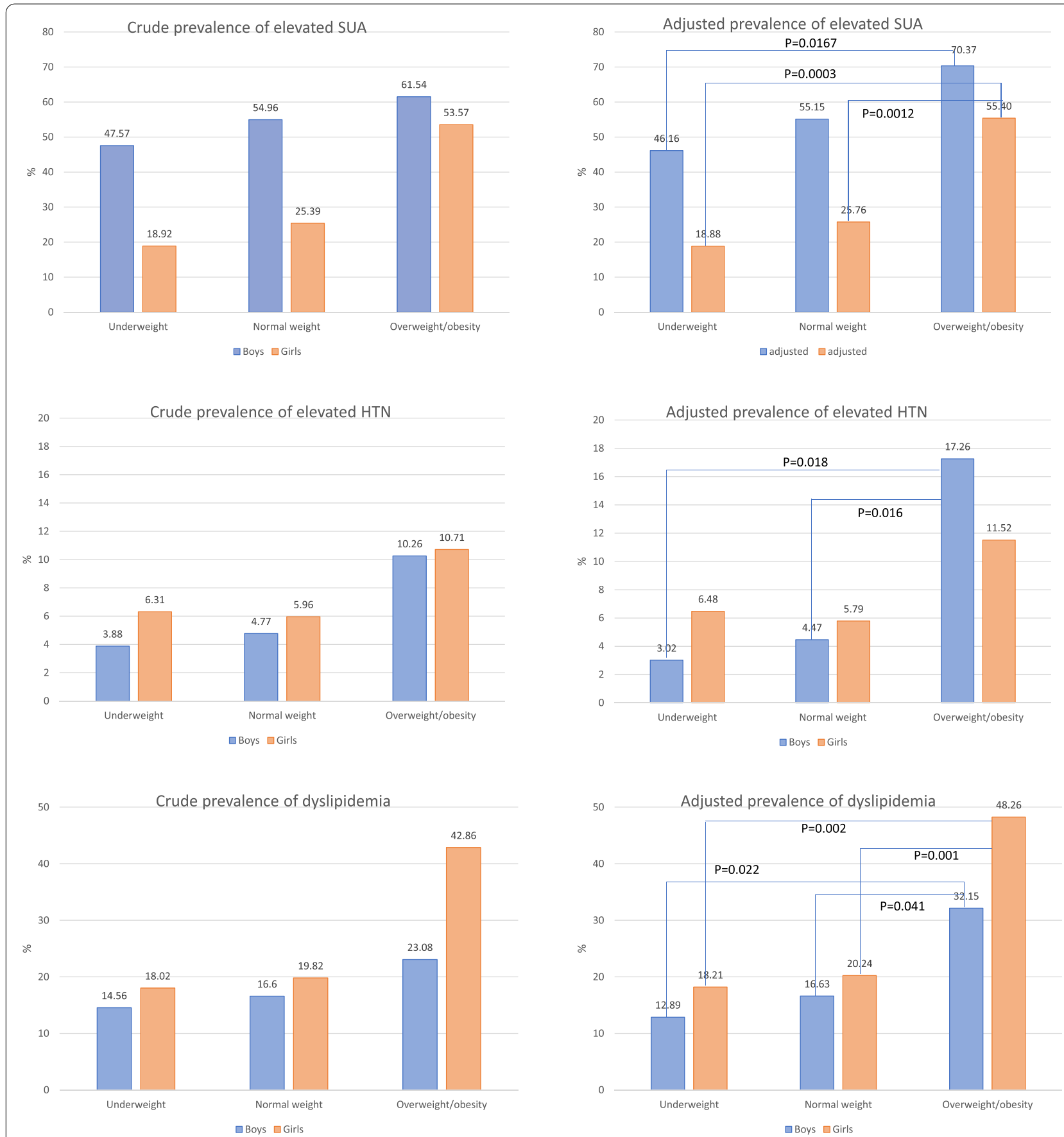

Fig. 3 Comparisons of cardiometabolic abnormalities between normal weight and overweight/obese children and adolescents aged 7-18. A The crude and adjusted prevalence of elevated SUA; B The crude and adjusted prevalence of HTN: The crude and adjusted prevalence of dyslipidemia. Covariates being adjusted were age and residential areas

atherosclerotic lesions in youth[31,32], and the elevated serum uric acid may further add to the burden of risk[33]. Obesity is a major cause of hyperuricemia in healthy children and adolescents without chronic conditions [34].
Our study revealed that, increased BMI was linked with elevated SUA, and this effect varies on sex. Boys had much higher SUA level than girls and the correlation between BMI and SUA was also stronger than girls. The negative association between SUA and BMI groups 


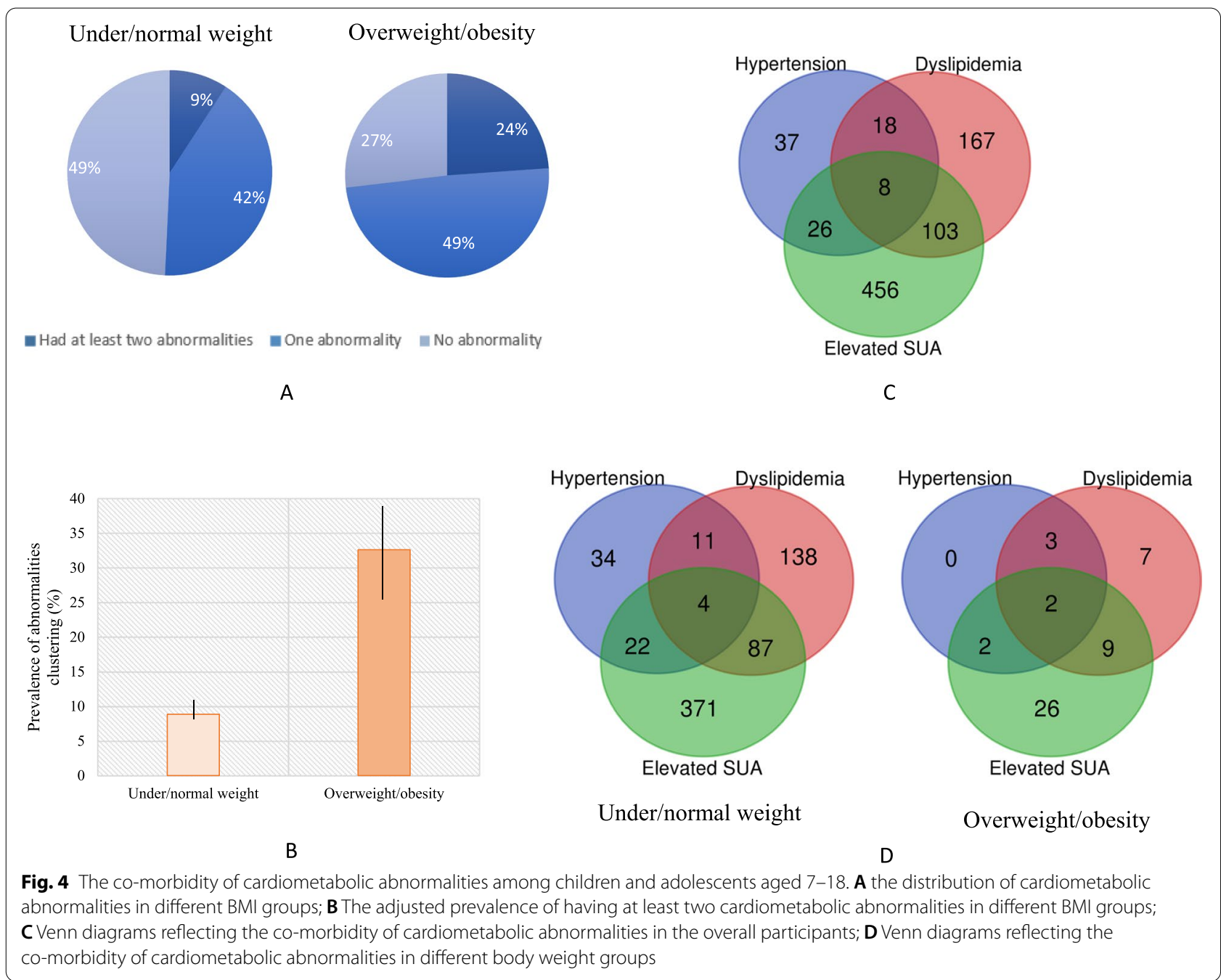

in boys may be due to the limited sample size and the cut-off value of serum uric acid. SUA and other metabolic components has complicated interactions. Elevated SUA can also influence other cardiometabolic risk factors and vice versa $[7,22,35]$. The mechanism of the linkage between BMI and uric acid may be explained by that dysfunction of obese adipose tissue could be associated with overproduction of uric acid[36]: increasing uric acid-dependent intracellular and mitochondrial oxidative stress, activating the nuclear transcription factor, carbohydrate responsive element-binding protein or inhibition of AMP-activated protein kinase [37-39].

Elevated body weight was also associated with increased risk of serum lipid disorders [18, 40], and this effect varies on sex. Girls had higher prevalence of dyslipidemia than boys, especially in the overweight/obese group ( $48.26 \%$ vs. $32.15 \%)$. Overweigh/obese girls were also more likely to suffer from dyslipidemia according to the logistic regression model. Although this sex disparity is inconsistent with the result revealed by NHANES for 1999-2006[41], which indicated a higher prevalence of dyslipidemia in boys, but a meta-analysis in China supported our conclusion in the same way of sex disparity [42]. Therefore, the difference may be attribute to diversity of genetic or socio-environmental factors. Recent studies indicated that triglycerides and triglyceride-rich lipoproteins are in the casual pathway for atherosclerotic cardiovascular disease, much like LDL-C $[43,44]$. The present study demonstrated that in both sex, higher TG and LDL-C was observed in the overweight/obese group. Although recent guidelines have not recommended lipid lowering therapy [45], it is important to initiate life-style interventions, such as weight loss, to reduce the hazard of developing further health damage.

Overweight and obesity are important risk factors for HBP in children $[17,46]$. In line with other studies [29, $47,48]$, the current study revealed that overweight/obese children and adolescents had higher BP levels, especially 
in boys. This partial correlation suggested that the relationship between BMI and SBP was stronger than with DBP. It implies that in the monitoring of high blood pressure in overweight or obese youths, SBP maybe more sensitive.

Few studies explored the clustering of cardiometabolic abnormalities. Seo et al. investigated the cardiovascular disease risk factors clustering (CVD-RFC) among Korean children and adolescents aged 6-15 [49], in which elevated $B P$ and serum lipids were considered as clustered factors also found the positive association between excess adiposity and CVD-RFC. Data from the Bogalusa Heart study [30] implied that over half of obese children had at least two cardiometabolic risk factors including adverse levels of serum lipids, insulin and blood pressure. As elevated serum uric acid has been considered as one of the most important risk factors of cardiovascular or cardiometabolic diseases, the clustering of risk factors including elevated SUA should be given more attention among youths. Our study indicated that in overweight/obese participants, $49 \%$ had one abnormality and $24 \%$ had at least two cardiometabolic abnormalities, much higher than in their counterparts. Among the cardiometabolic abnormalities, elevated SUA had a relatively high prevalence, even in the normal weight children and adolescents. Therefore, the early prevention of hyperuricemia should be considered as an important intervention target health issue in youths.

The investigation of co-morbidity of high blood pressure, serum lipids and SUA could provide more comprehensive estimation on disease risk and clues for risk factors prevention among children and adolescents. Several studies have reported the cardiovascular risk factors clustering in Chinese children and adolescents [25], but the measure of clustering is different with ours, thus make the comparison.

The strength of our study is the representative large sample size and the co-mobility estimation of cardiometabolic abnormalities clustering among children and adolescents. Given the limited data of cardiometabolic abnormalities clustering, especially the clustering of elevated SUA and other risk factors among Chinese children and adolescents, this study could provide evidence on disease burden estimation in youth in South China. In addition, in this study we used both linear and non-linear models to understand the association between excess adiposity and cardiometabolic abnormalities in multi-aspects. Nonetheless, the limitation of the current study should also be acknowledged. Firstly, using cross-sectional data, we cannot make causal inference between excess adiposity and cardiometabolic abnormalities. Given that both epidemiological and Mendelian randomization study have improved the causal role of overweight/obesity in the disease onset of cardiometabolic disorders $[50,51]$, and that intervention on body weight because of existed disease could result in an underestimation of the estimation, the association between body weight and cardiometabolic profiles were stable and helpful. Secondly, the lack of data on insulin resistance, dietary information, socialcultural and economic status information, and parental information limited our exploration.

In summary, with representative community-based sample, vigorous methodology, we find that excess adiposity increased the risk of elevated serum uric acid, serum lipids, blood pressure and their clustering among children and adolescents in a southernmost island of China. This study highlights the risk of overweight and/ or obesity on multiple cardiometabolic abnormalities and would be helpful for policy makers as well health practitioners to learn the current situation of local childhood disease burden and risk factors, and further useful for early intervention.

\section{Abbreviations}

BMI: Body mass index; Cl: Confidence interval; DBP: Diastolic blood Pressure; HDL-C: High-density lipoprotein cholesterol; IQR: Interquartile range; LDL-C: Low-density lipoprotein cholesterol; OR: Odds ratio; RCS: Restricted cubic spline; SBP: Systolic blood pressure; SD: Standard deviation; SUA: Serum uric acid; TC: Total cholesterol; TG: Triglycerides; WHO: World health organization.

\section{Acknowledgements}

This study was supported by Beijing Natural Science Foundation (7204279), the Key Basic Research Program of the Ministry of Science and Technology of China (Grant No. 2013FY114100), and National Natural Science Foundation of China (82003531). We would like to thank all the participants and staff members from Hainan and Shaanxi Provincial Centers for Disease Control and Prevention. We also gratefully appreciate Guangjin Zhu for her efforts and expertise in the field work.

\section{Authors' contributions}

Conception and design: HH, SG, PL, DJ; Administrative support: SG and PL; Provision of study materials or patients: DJ, JY, JP; Collection and assembly of data: HH, SG, PL, DJ, DJ, JY, JP; Data analysis and interpretation: HH, SG. Manuscript writing: $\mathrm{HH}$. All authors read and approved the final manuscript.

\section{Funding}

This study was supported by the Key Basic Research Program of the Ministry of Science and Technology of China (Grant No. 2013FY114100), Beijing Natural Science Foundation (7204279), and National Natural Science Foundation of China (82003531).

\section{Availability of data and materials}

The datasets generated and/or analyzed during the current study are not publicly available due to management rules by the study funder but are available from the corresponding author on reasonable request.

\section{Declarations}

Ethics approval and consent to participate

Ethics approval was obtained from the Bioethical Committee of Institute of Basic Medical Sciences, Chinese Academy of Medical Sciences. All methods were performed in accordance with the relevant guidelines and regulations (Declaration of Helsinki). Informed consent was obtained from parents/ 
guardians of the participants whose age was below 16 and informed consent was obtained from the participants whose age was above 16.

\section{Consent for publication}

Not appliable

\section{Competing interests}

The authors declare that they have no competing interests.

\section{Author details}

${ }^{1}$ Department of Epidemiology and Statistics, Institute of Basic Medical Sciences, Chinese Academy of Medical Sciences; School of Basic Medicine, Peking Union Medical College, 5 Dongdansantiao, Dongcheng District, Beijing 100005, China. ${ }^{2}$ Hainan Provincial Center for Disease Control and Prevention, Haikou 570203, China.

Received: 12 April 2021 Accepted: 22 December 2021 Published online: 10 January 2022

\section{References}

1. Raitakari OT, Juonala M, Kahonen M, Taittonen L, Laitinen T, Maki-Torkko $\mathrm{N}$, Jarvisalo MJ, Uhari M, Jokinen E, Ronnemaa T, et al. Cardiovascular risk factors in childhood and carotid artery intima-media thickness in adulthood: the Cardiovascular Risk in Young Finns Study. JAMA. 2003:290(17):2277-83.

2. Morrison JA, Friedman LA, Wang P, Glueck CJ. Metabolic syndrome in childhood predicts adult metabolic syndrome and type 2 diabetes mellitus 25 to 30 years later. J Pediatr. 2008;152(2):201-6.

3. Franks PW, Hanson RL, Knowler WC, Sievers ML, Bennett PH, Looker HC. Childhood obesity, other cardiovascular risk factors, and premature death. N Engl J Med. 2010;362(6):485-93.

4. Kjellberg E, Roswall J, Bergman S, Almqvist-Tangen G, Alm B, Dahlgren J. Longitudinal birth cohort study found that a significant proportion of children had abnormal metabolic profiles and insulin resistance at 6 years of age. Acta Paediatr. 2019;109(3):486-92.

5. Force UPST, Grossman DC, Bibbins-Domingo K, Curry SJ, Barry MJ, Davidson KW, Doubeni CA Jr, JWE, Kemper AR, Krist AH, et al. Screening for obesity in children and adolescents: US preventive services task force recommendation statement. JAMA. 2017;317(23):2417-26.

6. Rocha EPAA, Vogel M, Stanik J, Pietzner D, Willenberg A, Körner A, Kiess W. Serum uric acid levels as an indicator for metabolically unhealthy obesity in children and adolescents. Horm Res Paediat. 2018;90(1):19-27.

7. Genoni G, Menegon V, Secco GG, Sonzini M, Martelli M, Castagno M, Ricotti R, Monzani A, Aronici M, Grossini E, et al. Insulin resistance, serum uric acid and metabolic syndrome are linked to cardiovascular dysfunction in pediatric obesity. Int J Cardiol. 2017;249:366-71.

8. Lee EY, Yoon K. Epidemic obesity in children and adolescents: risk factors and prevention. Front Med. 2018;12(6):658-66.

9. Ng M, Fleming T, Robinson M, Thomson B, Graetz N, Margono C, Mullany EC, Biryukov S, Abbafati C, Abera SF. Global, regional, and national prevalence of overweight and obesity in children and adults during 1980-2013: a systematic analysis for the global burden of disease study 2013. Lancet. 2014;384(9945):766-81.

10. Guo $Y$, Yin $X$, Wu H, Chai $X$, Yang $X$. Trends in overweight and obesity among children and adolescents in China from 1991 to 2015: a metaanalysis. Int J Environ Res Public Health. 2019;16(23):4656.

11. $\mathrm{Ji} \mathrm{CY}$, Cheng TO. Epidemic increase in overweight and obesity in Chinese children from 1985 to 2005. Int J Cardiol. 2009;132(1):1-10.

12. Ashwell M, Gunn P, Gibson S. Waist-to-height ratio is a better screening tool than waist circumference and BMI for adult cardiometabolic risk factors: systematic review and meta-analysis. Obes Rev. 2012;13(3):275-86.

13. Lo K, Wong M, Khalechelvam P, Tam W. Waist-to-height ratio, body mass index and waist circumference for screening paediatric cardio-metabolic risk factors: a meta-analysis. Obes Rev. 2016;17(12):1258-75.

14. Zhang C. TSE L, Deng X, Jiang Z: Cardiovascular risk factors in overweight and obese Chinese children. Eur J Nutr. 2008;47(5):244-50.

15. LiY, Zou Z, Luo J, Ma J, Ma Y, Jing J, Zhang X, Luo C, Wang H, Zhao H, et al. The predictive value of anthropometric indices for cardiometabolic risk factors in Chinese children and adolescents: A national multicenter school-based study. PloS One. 2020;15(1):e227954.

16. Jiang Y, Dou YL, Xiong F, Zhang L, Zhu GH, Wu T, Zhang Y, Yan WL. Waist-to-height ratio remains an accurate and practical way of identifying cardiometabolic risks in children and adolescents. Acta Paediatr. 2018;107(9):1629-34.

17. He H, Pan L, Du J, Liu F, Jin Y, Ma J, Wang L, Jia P, Hu Z, Shan G. Body composition and serum total calcium were associated with blood pressure among children and adolescents aged 7-18 in China: a cross-sectional study. Front Pediatr. 2019;7:411.

18. He H, Pan L, Du J, Liu F, Jin Y, Ma J, Wang L, Jia P, Hu Z, Shan G. Prevalence of, and biochemical and anthropometric risk factors for, dyslipidemia in children and adolescents aged 7 to 18 years in China: A cross-sectional study. Am J Hum Biol. 2019;31(5):e23286.

19. World Health Organization: Overweight and Obesity.Aavailable at http:// www.who.int/news-room/fact-sheets/detail/obesity-and-overweight. Access on $14^{\text {th }}$ July, 2020.

20. Bamba V. Update on screening, etiology, and treatment of dyslipidemia in children. J Clin Endocrinol Metab. 2014;99(9):3093-102.

21. Flynn JT, Kaelber DC, Baker-Smith CM, Blowey D, Carroll AE, Daniels SR, de Ferranti SD, Dionne JM. Clinical practice guideline for screening and management of high blood pressure in children and adolescents. Pediatrics. 2017;140(3):e20171904.

22. Ford ES, Li C, Cook S, Choi HK. Serum concentrations of uric acid and the metabolic syndrome among US children and adolescents. Circulation. 2007;115(19):2526-32.

23. Roalfe AK, Holder RL, Wilson S. Standardisation of rates using logistic regression: a comparison with the direct method. BMC Health Serv Res. 2008:8:275

24. Durrleman S, Simon R. Flexible regression models with cubic splines. STAT MED. 1989;8(5):551-61.

25. Liu A, Hills AP, Hu X, Li Y, Du L, Xu Y, Byrne NM, Ma G. Waist circumference cut-off values for the prediction of cardiovascular risk factors clustering in Chinese school-aged children: a cross-sectional study. BMC Public Health. 2010;10:82.

26. Wang S, Dong Y, Wang Z, Zou Z, Ma J. Trends in overweight and obesity among Chinese children of 7-18 years old during 1985-2014. Chin J Prev Med. 2017;51(04):300-5 ((in Chinese)).

27. Cheng H, Chen FF, Ye PY, Mi J. Characteristics of cardiometabolic risk factors of children and adolescents aged 6-17 years in seven cities in China from 2013 to 2015. Chin J Prev Med. 2018;52(11):1130-5 ((in Chinese)).

28. Tong S, Zhou L, Zhang H, Li X, Yang D, Zhang W, Tian W, Zhao Y, Sun D, Yang B. Prevalence of hypertension in cold and non-cold area of China: a meta-analysis. Chin J Endemiol. 2018;37(5):420-5.

29. Zhang YX, Zhao JS, Chu ZH. The relationship of body mass index to blood pressure levels among children and adolescents in 30 provinces in China. Int J Cardiol. 2016;202:512-3.

30. Freedman DS, Mei Z, Srinivasan SR, Berenson GS, Dietz WH. Cardiovascular risk factors and excess adiposity among overweight children and adolescents: the bogalusa heart study. J Pediatr. 2007;150(1):12-7.

31. Celermajer DS, Sorensen KE, Gooch VM, Spiegelhalter DJ, Miller OI, Sullivan ID, Lloyd JK, Deanfield JE. Non-invasive detection of endothelial dysfunction in children and adults at risk of atherosclerosis. Lancet. 1992;340(8828):1111-5.

32. Ruiz LD, Zuelch ML, Dimitratos SM, Scherr RE. Adolescent obesity: diet quality, psychosocial health, and cardiometabolic risk factors. Nutrients. 2019;12(1):43.

33. Tin A, Marten J, HalperinKuhns VL, Li Y, Wuttke M, Kirsten H, Sieber KB, Qiu C, Gorski M, Yu Z, et al. Target genes, variants, tissues and transcriptional pathways influencing human serum urate levels. Nat Genet. 2019;51(10):1459-74.

34. Kubota M. Hyperuricemia in children and adolescents: present knowledge and future directions. J Nutr Metab. 2019;2019:3480718.

35. Sun D, Li S, Zhang X, Fernandez C, Chen W, Srinivasan SR, Berenson GS. Uric acid is associated with metabolic syndrome in children and adults in a community: the Bogalusa Heart Study. PloS One. 2014;9(10):e89696.

36. Tsushima Y, Nishizawa H, Tochino Y, Nakatsuji H, Sekimoto R, Nagao H, Shirakura T. Uric acid secretion from adipose tissue and its increase in obesity. J Bio Chem. 2013;288(38):27138-49.

37. Lanaspa MA, Sanchez-Lozada LG, Choi YJ, Cicerchi C, Kanbay M, RoncalJimenez CA, Ishimoto T, Li N, Marek G, Duranay M, et al. Uric acid induces 
hepatic steatosis by generation of mitochondrial oxidative stress: potential role in fructose-dependent and -independent fatty liver. J BIOL CHEM. 2012;287(48):40732-44.

38. Johnson RJ, Nakagawa T, Sanchez-Lozada LG, Shafiu M, Sundaram S, Le M, Ishimoto T, Sautin YY, Lanaspa MA. Sugar, uric acid, and the etiology of diabetes and obesity. Diabetes. 2013;62(10):3307-15.

39. Lanaspa MA, Cicerchi C, Garcia G, Li N, Roncal-Jimenez CA, Rivard CJ, Hunter B, Andres-Hernando A, Ishimoto T, Sanchez-Lozada LG, et al. Counteracting roles of AMP deaminase and AMP kinase in the development of fatty liver. PloS One. 2012;7(11):e48801.

40. Parray IA, Parry MA, Latief M. Prevalence of dyslipidemia in school children of Kashmir valley. Diabetes Metab Syndr. 2016;10(2 Suppl 1):S47-54.

41. Center for Disease Control and Prevention. Prevalence of abnormal lipid levels among youths -- United States, 1999-2006. MMWR-Morb Mortal Wkly Rep. 2010;59(2):29-33.

42. Ding W, Dong H, Mi J. Prevalence of dyslipidemia in Chinese children and adolescents:a meta-analysis. Chin J Epidemiol. 2015;36(1):71-7 ((in (hinese)).

43. Budoff M. Triglycerides and triglyceride-rich lipoproteins in the causal pathway of cardiovascular disease. Am J Cardiol. 2016:118(1):138-45.

44. Varbo A, Benn M, Tybjaerg-Hansen A, Nordestgaard BG. Elevated remnant cholesterol causes both low-grade inflammation and ischemic heart disease, whereas elevated low-density lipoprotein cholesterol causes ischemic heart disease without inflammation. Circulation. 2013;128(12):1298-309.

45. Expert panel on integrated guidelines for cardiovascular health and risk reduction in children and adolescents: summary report. Pediatrics. 2011;128(Suppl 5):S213-56.

46. Rao G. Diagnosis, epidemiology, and management of hypertension in children. Pediatrics. 2016;138(2):e20153616.

47. Kit BK, Kuklina E, Carroll MD, Ostchega Y, Freedman DS, Ogden CL. Prevalence of and trends in dyslipidemia and blood pressure among US children and adolescents, 1999-2012. JAMA Pediatr. 2015;169(3):272-9.

48. Wang SR, Zhu YB, Cheng Y, Zhang YX. Profiles of blood pressure among children and adolescents with different body mass index categories in Shandong China. Blood Press. 2018;27(1):56-61.

49. Seo Y, Choi M, Kang J, Lee H, Jang HB, Park SI, Ju YS, Park KH. Cardiovascular disease risk factor clustering in children and adolescents a prospective cohort study. Arch Dis Child. 2018;103(10):968-73.

50. Farmer RE, Mathur R, Schmidt AF, Bhaskaran K, Fatemifar G, Eastwood SV, Finan C, Denaxas S, Smeeth L, Chaturvedi N. Associations between measures of sarcopenic obesity and risk of cardiovascular disease and mortality: a cohort study and mendelian randomization analysis using the UK Biobank. J Am Heart Assoc. 2019;8(13):e11638.

51. Jansen H, Samani NJ, Schunkert H. Mendelian randomization studies in coronary artery disease. Eur Heart J. 2014;35(29):1917-24.

\section{Publisher's Note}

Springer Nature remains neutral with regard to jurisdictional claims in published maps and institutional affiliations.

Ready to submit your research? Choose BMC and benefit from:

- fast, convenient online submission

- thorough peer review by experienced researchers in your field

- rapid publication on acceptance

- support for research data, including large and complex data types

- gold Open Access which fosters wider collaboration and increased citations

- maximum visibility for your research: over $100 \mathrm{M}$ website views per year

At BMC, research is always in progress.

Learn more biomedcentral.com/submissions 